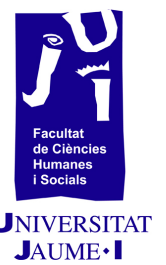

\title{
Relaciones entre la memoria de trabajo y la adquisición del lenguaje en alumnado con implante coclear en edad preescolar:
}

orientaciones educativas

\author{
Marta Boronat Barbeta \\ mboronat1@gmail.com \\ Rosana Clemente Estevan \\ clemente@psi.uji.es \\ Nuria Silvestre Benach \\ nuria.silvestre@uab.cat
}


I. Resumen

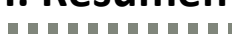

El trabajo se centra en un estudio preliminar sobre el nivel de memoria de trabajo en niños y niñas con desarrollo típico y con implante coclear (IC), debido a su relación con el lenguaje. Los resultados evidencian las carencias en los diferentes niveles de la habilidad ejecutiva de este tipo de memoria en los niños y las niñas con IC, así como la necesidad de realizar una intervención temprana para reforzar su desarrollo y favorecer el lenguaje. Se presentan diferentes actividades que promueven la estimulación de esta área.

Palabras clave: implante coclear, memoria de trabajo, desarrollo lingüístico, intervención temprana, función ejecutiva.

\section{Introducción}

Las criaturas con hipoacusia profunda o severa prelocutiva precisan de un implante coclear para recibir los sonidos del entorno. La mayoría de ellas, gracias a los avances de la tecnología médica, aprenden el lenguaje oral expresivo y comprensivo con un nivel próximo a sus iguales. Sin embargo, estas ventajas no beneficia a todos los niños por igual, puesto que el rendimiento del implante varía en función de cada usuario. Esta inestabilidad se trata de subsanar mediante el apoyo de rehabilitación logopédica junto con los agentes educativos (la familia y la escuela), que son considerados coparticipantes de algunos aspectos en la adquisición del lenguaje.

Una posible razón de las dificultades que persisten en la adquisición del lenguaje en los infantes sordos, teniendo en cuenta unas condiciones óptimas (temprana edad, modo de comunicación oral, apoyo logopédico, implicación de los padres, etc.) es que la falta de desarrollo del lenguaje acorde a la edad y las experiencias auditivas tempranas afecten a las funciones ejecutivas (FE) (Pisoni et al., 2010).

Las FE son habilidades cognitivas capaces de desarrollar el control sobre la propia conducta, la actividad emocional y cognitiva. La memoria de trabajo es la capacidad de memorizar y de codificar los procesos de mantenimiento de la información, estableciendo algún tipo de interferencia o de consigna que obligue a reelaborar la información relevante. Concretamente, las investigaciones exponen que hay cuatro habilidades vinculadas al desarrollo del lenguaje y el habla: la memoria de trabajo, la velocidad en la fluidez del habla, la concentración-atención-inhibición y la organización-integración (Pisoni et al., 2010). Pisoni y sus colaboradores opinan que la lengua oral se emite $y$ tiene que procesarse por los oyentes muy rápidamente en unidades representativas que deben ser 
reconocidas, organizadas, integradas $y$, finalmente, almacenadas en

la memoria a corto (memoria de trabajo) y a largo plazo, manteniendo siempre un alto nivel de atención. La memoria de trabajo es, por tanto, fundamental en este proceso, puesto que las unidades lingüísticas se deben recordar en su organización y secuencia para que el mensaje sea comprendido (Cleary, Pisoni, y Geers, 2001; Pisoni et al., 2010).

La memoria de trabajo es una habilidad que inicia su desarrollo en las primeros años del niño; por tanto, tienen una gran peso en el aprendizaje del lenguaje durante la etapa de educación infantil e inicio de la etapa de educación primaria (Röthlisberger, Neuenschwander, Cimeli, Michel y Roebers, 2011). A este respecto, Vissers, Koolen, Hermans, Scheper y Knoors (2015) mostraron que las criaturas en edad preescolar con retrasos severos en el desarrollo del habla y el lenguaje mostraban menor formación en memoria de trabajo, así como en otras $\mathrm{FE}$, como la inhibición y la flexibilidad cognitiva. En particular, se ha evidenciado que existe una intima relación entre la habilidad de memoria de trabajo con el lenguaje en niños y niñas con hipoacusia y con desarrollo típico (Kronenberger, Colson, Henning y Pisoni, 2014)

En relación con esta misma línea de investigación, los niños con IC presentan un alto riesgo en el desarrollo de las habilidades ejecutivas de memoria de trabajo, fluidez del habla concentración e inhibición (Conway y Kronenberger 2009; Kronenberger et al., 2013). En un trabajo reciente, Beer et al. (2014) encontraron en 24 participantes sordos con IC de entre 3-6 años peor rendimiento en tareas sobre control inhibitorio y memoria de trabajo en comparación con su grupo de iguales. Además, el déficit en la memoria de trabajo se mantiene en edades posteriores según un estudio de Pisoni y Cleary (2003) en el que observaron en criaturas de 8-9 años de edad con IC que mostraban diferencias significativas en la extensión de los dígitos hacía delante, prueba de memoria de trabajo verbal a corto plazo, en comparación con sus compañeros normoyentes. Además, la puntuaciones de memoria de trabajo se correlacionaron significativamente con las puntuaciones de reconocimiento de palabras habladas. Estos hallazgos se replican en un estudio realizado por Burkholder y Pisoni (2003), en el que también se observa que los niños sordos con IC tuvieron una duración mayor de la pausa en la recuperación de los dígitos que menciona el examinador, por lo que refleja un proceso lento en el uso de estrategias de recuperación de la información fonológica, así como otros mecanismos de recuerdo, dado que la prueba de dígitos hacia atrás correlaciona con el habla en los niños con IC, pero no con los niños con desarrollo típico. De este modo, podemos considerar que los niños sordos, ante la ausencia de experiencia auditiva temprana e integración del proceso fonológico previos a la 
implantación, perjudica el desarrollo de la memoria de trabajo (Burkholder y Pisoni, 2003), y presenta limitaciones en la adquisición de mecanismos de procesamiento para codificar y mantener las secuencias de dígitos, los cuales ya dominan los niños oyentes a esas edades.

Todavía debemos profundizar más en el campo de las funciones ejecutivas de la población con hipoacusia en la edad infantil para conocer el impacto en el desarrollo de las habilidades lingüísticas, pero las evidencias anteriores ponen de manifiesto que la memoria de trabajo y las habilidades lingüísticas se relacionan de manera distinta en niños con IC en comparación con normoyentes. Por ello, se hace necesario realizar estudios centrados en la intervención sobre las habilidades cognitivas para conocer sus beneficios en el aprendizaje.

Hasta el momento se han realizado pocos estudios de intervención. Kronenberger et al. (2011) estableció un programa de intervención durante un mes y observó que los participantes con IC de 7-15 años mejoraron significativamente en memoria de trabajo y dicha mejora se mantuvo un mes después de la estimulación. Además, las puntuaciones brutas de repetición de oraciones mejoraron significativamente sobre las anteriores a la realización del programa y continuaron esa tendencia después de seis meses. No obstante, aunque el grupo de participantes sea de edad escolar y los estudios sean minoritarios, se demuestra la posibilidad de mejorar de manera significativa las habilidades cognitivas en alumnado con IC.

\section{Objetivos}

Aportar conocimiento científico sobre el nivel de desarrollo de la habilidad cognitiva de la memoria de trabajo en el proceso de aprendizaje de las habilidades lingüísticas de la población infantil con desarrollo típico y con implante coclear (IC), y dotar de orientaciones educativas para la estimulación del área de estudio, a fin de paliar los déficits que se presenten.

\section{Material y método}

Los participantes han sido seleccionados de un centro educativo de atención preferente a alumnado con sordera. Se escogió el grupoclase del segundo ciclo de educación infantil, debido al mayor número de participantes con hipoacusia. El número total de participantes eran 13 , de los cuales diez (siete niños y tres niñas) eran alumnos con desarrollo típico y tres (dos niños y una niña), con hipoacusia prelocutiva bilateral profunda, usuarios de implante 
coclear. Los tres estudiantes recibieron el implante antes de los 28 meses y la ganancia prevista oscilaba entre los 50 y los 60 decibelios.

Los instrumentos de evaluación que se pasaron a todos los participantes fueron los siguientes: en primer lugar, Escala de Desarrollo del Lenguaje de Reynell (1997), que evalúa la comprensión y expresión oral. El test consta de 124 ítems (62 por cada escala). En segundo lugar, Tarea de dígitos: Wechsler Intelligence Scale for Children - IV (WISC-IV), que se centra en la evaluación de la memoria de trabajo mediante la expresión de dígitos hacia delante y hacia atrás.

El procedimiento que se siguió para la consecución del objetivo de estudio fue la realización de una reunión informativa en el centro educativo sobre el proyecto de estudio. Posteriormente, se envió un documento informativo a las familias, así como la solicitud del permiso del centro y los padres o tutores de los participantes para poder evaluar el área de la memoria de trabajo, entre otras FE.

Para realizar las comparaciones se utilizó estadística no paramétrica $U$ de Mann-Whitney de comparación entre dos muestras independientes. Se usó el programa SPSS versión 24.

\section{Resultados}

Tabla 1. Memoria de trabajo y lenguaje. Media, desviación típica, rango y su significación en los grupos de participantes sordos y oyentes

\begin{tabular}{|l|l|l|l|l|l|l|}
\hline Tareas & \multicolumn{2}{|l|}{ Sordos (N=3) } & \multicolumn{2}{l|}{ Oyentes (N=10) } & & \\
\hline & M (DT) & Rango & M (DT) & Rango & U & $\mathrm{p}$ \\
\hline $\begin{array}{l}\text { Memoria } \\
\text { trabajo }\end{array}$ & $2,67(0,57)$ & $2-3$ & $\begin{array}{l}7,1 \\
(2,07)\end{array}$ & $4-10$ & 0,000 & $0,007^{* *}$ \\
\hline $\begin{array}{l}\text { Lenguaje } \\
\text { comprensivo }\end{array}$ & $30(5,29)$ & $24-34$ & $\begin{array}{l}52,60 \\
(3,62)\end{array}$ & $48-59$ & 0,000 & $0,007^{* *}$ \\
\hline $\begin{array}{l}\text { Lenguaje } \\
\text { expresivo }\end{array}$ & $14(8,38)$ & $9-24$ & $\begin{array}{l}39,60 \\
(3,43)\end{array}$ & $34-44$ & 0,000 & $0,007^{* *}$ \\
\hline
\end{tabular}


Tabla 2. Correlaciones entre las tareas evaluadas sobre las subáreas de la memoria en relación con el lenguaje expresivo y comprensivo de todo el grupo de participantes

\begin{tabular}{|c|c|c|}
\hline \multicolumn{1}{|c|}{ Tareas } & Lenguaje comprensivo & Lenguaje expresivo \\
\hline \multicolumn{1}{|c|}{ Memoria trabajo } & $0,728^{* *}$ & $0,754^{* *}$ \\
\hline $\begin{array}{l}\text { Lenguaje } \\
\text { comprensivo }\end{array}$ & 1 & $0,931^{* *}$ \\
\hline \multicolumn{2}{|c}{$* \mathrm{p}<0,05, * * \mathrm{p}<0,01$} \\
\hline
\end{tabular}

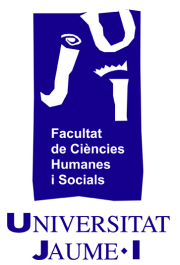

\section{Discusión y conclusiones}

Las diferencias entre los grupos de participantes son claras, como demostraron también los estudios previos de Kronenberger et al. (2014) y Lyxell et al. (2008). Por tanto, se evidencia la íntima correlación que existe entre ambas áreas (lenguaje y memoria de trabajo).

Las evidencias de los resultados señalan la necesidad de establecer un programa de estimulación en el área de la memoria en general y de trabajo en particular sobre el alumnado preescolar con hipoacusia, con el propósito de reducir los déficits.

Los hallazgos relevantes de este estudio residen en el registro de datos de las carencias visibles en el desarrollo de un subtipo muy relevante de la memoria en el período de edad preescolar, en consecuencia, precisan de un programa de atención temprana en el que se realice la estimulación en esta área cognitiva para paliar las dificultades en el aprendizaje.

La limitación de este estudio es el grupo minoritario de participantes sordos con IC, pero este estudio preliminar muestra resultados coincidentes con los datos de la bibliografía.

Las orientaciones educativas para disminuir el nivel bajo en el área de la memoria se deben plantear desde una visión inclusiva, en la que sean partícipes todos los alumnos del aula de educación infantil. Las actividades se pueden plantear de manera grupal o por parejas; en estos casos es recomendable formarlas por un participante con hipoacusia y un compañero/a con desarrollo típico, a fin de mantener un enfoque ecológico y participativo. Por ello, las actividades deben ser descritas previamente al docente para que puedan trabajar de manera conjunta y así obtener un mayor éxito en la intervención.

La estimulación de la memoria de trabajo debe realizarse mediante actividades dinámicas con una dificultad gradual, estableciendo la consecución progresiva de los objetivos, y favorecer un aprendizaje experiencial educativo e inclusivo. Principalmente las dificultades se observan en la ejecución de una atención dividida, donde se debe atender a la discriminación de los sonidos y el mantenimiento mediante estrategias de una información para recuperarla con la ejecución de la orden correspondiente, como, por 
ejemplo, la actividad denominada «Tres números, tres acciones»: La actividad consiste en tres números que escogemos y a cada uno se le otorga una acción que deben ejecutar los participantes cuando lo escuchen; así, deben estar atentos al número que se menciona y recordar la acción que deben realizar para ejecutarla. De este modo, si previamente otorgamos al número uno la acción de saltar, cuando se mencione solo este número, los participantes deben ejecutar la acción. Además, este ejercicio interviene en otras FE, como la atención e inhibición.

Otra actividad sería aplicar el juego BrainBox; este juego consiste en atender a la imagen durante 10 segundos y ocultarla para realizar las preguntas, que aparecen en el dorso de la imagen, sobre detalles de la imagen. La dificultad en los alumnos sordos es el tiempo reducido para atender y recordar detalles tanto generales como concretos de la imagen, porque desconocen las preguntas. Por ello, es conveniente ampliar el tiempo de atención a la imagen, aproximadamente 25 segundos más, establecer preguntas cerradas (sí/no) y escoger imágenes sencillas. Progresivamente se escogerán imágenes más complejas, se reducirá el tiempo y, en última instancia, se ampliarán los modelos de preguntas.

Este estudio preliminar nos permite constatar que se puede evaluar la función ejecutiva de la memoria de trabajo en edad preescolar y obtener datos relevantes sobre el aprendizaje del alumnado con sordera. En este caso, a pesar de la inclusión total del alumnado con hipoacusia y el apoyo tecnológico del implante coclear, se ha observado que a la edad de cuatro años de edad ya se muestra un déficit en el área de la memoria que precisa una intervención temprana en esta área, sobre todo por su interrelación en el desarrollo de las habilidades lingüísticas.

Finalmente, los estudios sobre intervención nos abren las puertas a nuevas oportunidades de estudio sobre los beneficios de las actividades de juego grupales o individuales en el aprendizaje y su eficacia en la reducción de los perjuicios a corto y largo plazo.

\section{Bibliografía}

Beer, Jessica, William G. Kronenberger, Irina Castellanos, Bethany G. Colson, Shirley C. Henning y David Pisoni. 2014. «Executive Functioning Skills in Preschool-Age Children With Cochlear Implants». Journal of Speech, Language, and Hearing Research 57: 1521-1534.

Burkholder, Rose y David Pisoni. 2003. «Speech Timing and Working Memory in Profoundly Deaf Children after Cochlear Implantation». Journal of Experimental Child Psychology 85 (1): 63-88. 
Cleary, Miranda, David Pisoni y Ann Geers. 2001. «Some Measures of Verbal and Spatial Working Memory in Eight- and Nine-Year-Old Hearing-Impaired Children with Cochlear Implants». Ear and Hearing 22 (5): 395-411.

Conway, Christopher y William Kronenberger. 2009. «The Importance of Sound for Cognitive Sequencing Abilities: The Auditory Scaffolding Hypothesis». Current Directions in Psychological Science 18: 275-279.

Kronenberger, William, David B. Pisoni, Shirley C. Henning, Bethany G. Colson y Lindsey M. Hazzard. 2011. «Working Memory Training for Children With Cochlear Implants: A study». Journal of Speech, Language, and Hearing Research 54 (4): 1182-1196.

Kronenberger, William, David B. Pisoni y Shirley C. Henning. 2013. "Executive Functioning Skills In Long-Term Users of Cochlear Implants: A case Control Study». Journal of Pediatric Psychology 38 (2): 902-914.

Kronenberger, William, Bethany G. Colson, Shirley C. Henning y David B. Pisoni. 2014. "Executive Functioning and Speech-Language Skills Following Long-Term Use of Cochlear Implants». Journal of Deaf Studies and Deaf Education 19 (4): 456-470.

Lyxell, Björn, Birgitta Sahlén, Malin Wass, Tina Ibertsson, Birgitta Larsby, Mathias Hällgren y Elina Mäki-Torkko. 2008. "Cognitive Development in Children with Cochlear Implants: Relations to Reading and Communication». International Journal of Audiology 47 (2): 547-552.

Pisoni, David y Miranda Cleary. 2003. «Measures of Working Memory Span and Verbal Rehearsal Speed in Deaf Children after Cochlear Implantation». Ear Hear 24: 1-23.

Pisoni, David, Christopher M. Conway, William G. Kronenberger, Shirley Henning y Esperanza Anaya. 2010. «Executive Function, Cognitive Control, and Sequence Learning in Deaf Children with Cochlear Implants». En The Oxford Handbook of Deaf Studies, Language, and Education, vol. 2, editado por Mark Marschark y Patricia Elizabeth Spencer. Nueva York: Oxford University Press.

Röthlisberger, Marianne, Regula Neuenschwander, Patriza Cimeli, Eva Michel y Claudia M. Roebers. 2011. «Improving executive functions in 5- and 6-year-olds: Evaluation of a small group intervention in prekindergarten and kindergarten children". Infant and Child Development 21 (4): 411-429.

Vissers, Constance, Sophieke Koolen, Daan Hermans, Annette Scheper y Harry Knoors. 2015. «Executive Functioning in Preschoolers with Specific Language Impairment». Frontiers in Psychology 6: 1574. 